\title{
Low frequency impedance spectroscopy analysis of thermoelectric modules
}

\author{
Jorge García-Cañadas* and Gao Min \\ Cardiff School of Engineering, Cardiff University, The Parade, Cardiff, CF24 3AA, United \\ Kingdom
}

Phone: +44 (0)2920 875950

Fax: +44 (0)2920 874939

* Corresponding author: garciacanadasj@cardiff.ac.uk

\begin{abstract}
Impedance spectroscopy is a well-established technique for the study of semiconductors and energy related devices. However, in the area of thermoelectrics (TEs) is not frequently used and there is a lack of a physical background for a proper interpretation of the results. Usually, in the low frequency regime the impedance spectrum of TE modules working in cooling mode is characterized by a semicircle which can be modelled as a parallel connection of a resistor and a capacitor. Here we present a theoretical analysis to understand the origin of both parameters in bulk thermoelectric modules working as Peltier coolers. The analysis introduces a thermoelectric capacitance and a thermoelectric resistance that are defined by the temperature, the Seebeck coefficient and the thermal properties of the module (specific heat and thermal conductivity respectively). The product of both provides a time constant that directly relates to the thermal diffusivity. Our analysis provides a theoretical model able to interpret the low frequency results and obtain relevant thermal parameters from a single impedance measurement.
\end{abstract}

Keywords Characterisation of thermoelectric modules, ac electrical impedance. thermal constants

\section{Introduction}

Impedance spectroscopy (IS) is a powerful technique extensively used for characterisation of a wide range of electronic materials and devices (i. e., solar cells [1], fuel cells [2], photoelectrochemical hydrogen generation [3], batteries and supercapacitors [4]). It has the ability to separate in the frequency domain most of the processes that govern the performance of the 
devices, providing useful insight and quantitative measure of the physical processes of the system. However, it requires suitable physical models for correct interpretation of the equivalent circuit obtained by appropriate fitting of measurement data. In the thermoelectric (TE) field this technique is not frequently used [5-11] and it lacks a suitable physical model able to provide all the information contained in the impedance spectra.

Measurements performed on TE modules show a characteristic semicircle at low frequencies in the Nyquist representation which can be modelled as a parallel connection of a resistor and a capacitor. The resistance is in the order of few $\Omega$ and the capacitance is about 1 F. This value of the capacitance is quite large compared to conventional capacitors [11] and its origin is far from possible contact capacitances that could lie in the range of $\mu \mathrm{F}$. In order to obtain a physical model for a proper interpretation of the impedance results in TEs it is important to understand the physical meaning of this capacitance and the related resistance. In this paper we present an analysis to determine the origin of these parameters. The new concepts of a thermoelectric capacitance and a thermoelectric resistance are introduced. A physical meaning and definition is provided. Furthermore, a time constant determined by the product of both allows the direct determination of the thermal diffusivity. This knowledge will provide a starting point for the proper understanding of the impedance results in TEs, gaining insights into a whole physical picture of the devices under actual working conditions.

\section{Experimental part and discussion}

A commercial TE module (European Thermodynamics Ltd., Leicester, England) formed by 254 squared thermoelements $\left(1 \times 1 \times 1.5 \mathrm{~mm}^{3}\right)$ was suspended in vacuum $\left(\approx 10^{-5}\right.$ torr $)$ and connected to an Autolab PGSTAT 302N potentiostat equipped with a FRA32M impedance module (Metrohm Autolab B. V., Utrecht, The Netherlands). Impedance measurements were performed at room temperature at $0 \mathrm{~V}$ dc with a $10 \mathrm{mV}$ amplitude from $1 \mathrm{mHz}$ to $1 \mathrm{MHz}$. 


\section{Theoretical results}

The solution to the steady state heat balance equation for an $n$-type thermoelement assuming non-temperature dependence of the TE properties provides the heat removal rate at the cold side as [12-13],

$$
Q_{C}=\pi I-\frac{\lambda A}{L} \Delta T-\frac{1}{2} I^{2} R
$$

where $Q_{C}$ is the heat flow extracted from the cold side, $A$ is the cross-sectional area, $L$ the element length, $\pi$ the Peltier coefficient, $I$ the electric current, $\lambda$ the thermal conductivity, $\Delta T$ the temperature difference and $R$ is the ohmic resistance of the thermoelement. For a TE module suspended in vacuum, it is assumed that $Q_{C}=0$, then,

$$
0=\pi I-\frac{\lambda A}{L} \Delta T-\frac{1}{2} I^{2} R
$$

The temperature difference can be obtained from Eq. 2 as,

$$
\Delta T=\frac{L}{\lambda A}\left(\pi I-\frac{1}{2} I^{2} R\right)
$$

We define a TE resistance $R_{T E}$ from the voltage contribution due to the TE effects that exists in the total voltage $V$ applied to the thermoelement, $V=\alpha \Delta T+I R$,

$$
R_{T E}=\frac{\Delta V_{\alpha}}{I}=\frac{\alpha}{I} \Delta T
$$

where $\Delta V_{\alpha}=V(x=0)-V(x=L)$. This resistance relates to the energetic losses that the carriers experience by the electric field created by the temperature difference (Seebeck voltage). Introducing Eq. 3 in Eq. 4, we obtain,

$$
R_{T E}=\frac{\alpha L}{\lambda A}\left(\pi-\frac{1}{2} I R\right)
$$

This equation has units of $\Omega$ and relates to the TE parameters of the device. A large value for $R_{T E}$ is obtained for small $\lambda$. 
Alternatively, we define a thermoelectric capacitance $C_{T E}$ as,

$$
C_{T E}=I \frac{d t}{d V_{\alpha}}=\frac{I}{\alpha}\left(\frac{d T}{d t}\right)^{-1}
$$

where $V_{\alpha}$ is the Seebeck voltage. The physical meaning of $C_{T E}$ is not completely clear from the above equation since it could be related to either the charge accumulation (Idt) due to the reorganization of the carriers or the rate at which the temperature gradient is built $(d T / d t)$. We will try to clarify this in a future work. By looking at the thermal diffusivity $\beta$, which depends on the thermal conductivity, the material's density $\rho$ and the specific heat $C_{p}$,

$$
\beta=\frac{\lambda}{\rho C_{p}}
$$

and using the Fourier's law of heat conduction $(d Q / A d t=-\lambda d T / d x)$ and the definition of the specific heat $\left(C_{p}=d Q / \rho A L d T\right)$, we obtain,

$$
d t=\frac{-L}{\beta} d x
$$

We should remark that this equation is only correct when the variation of the conductive heat flux is only due to the heat absorption in the material, i. e., when the Joule effect is neglected. If we introduce Eq. (8) in Eq. (6), it results,

$$
C_{T E}=\frac{-I L}{\alpha \beta}\left(\frac{d T}{d x}\right)^{-1}
$$

Finally, using the heat balance at steady state at the cold junction,

$$
\left(\frac{d T}{d x}\right)^{-1}=\frac{-\lambda A}{I \pi}
$$

we obtain,

$$
C_{T E}=\frac{\rho C_{p} A L}{\alpha \pi}
$$


This equation has units of $\mathrm{F}$ and as occurred for $R_{T E}$ is a function of the TE properties of the thermoelement. It shows that the capacitance of the device increases with the specific heat. In a TE module working as a Peltier cooler, the external applied voltage $V_{d}$ sets an ohmic current $I_{d}$ through the circuit as shown in Fig. 1. The higher the current the higher the cooling effect. However, when a temperature gradient starts to rise due to the Peltier effect, the carriers reorganize and the Seebeck voltage appears, which creates the inverse current $I_{\alpha}$ (Fig. 1).

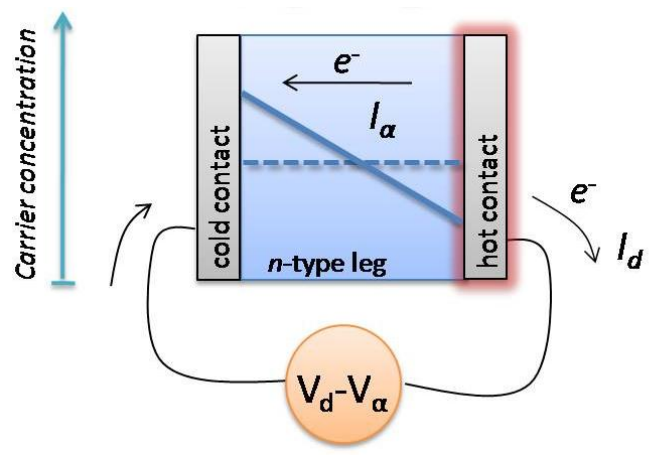

Figure 1 Scheme to illustrate the TE module operation as Peltier cooler. In equilibrium (no voltage applied), the carrier concentration is constant along the material (dashed line). However, when a current is applied the Peltier effect takes place and the carriers reorganize/accumulate (solid line) due to the temperature gradient appearing.

Both equations obtained for $C_{T E}$ and $R_{T E}$ are in agreement with the results from Downey et al. [8] when neglecting the Joule effect. However, unlike these authors, we have included here the influence of the Joule effect in $R_{T E}$ and the derivation of the parameters from physical definitions instead of using a direct comparison between thermal and electrical circuits.

By multiplying both $R_{T E}$ and $C_{T E}$ (neglecting Joule heating) we obtain a time constant $\tau$,

$$
\tau=R_{T E} C_{T E}=\frac{L^{2}}{\beta}
$$

defined by the thermal diffusivity, which determines how fast heat is transported while it is been absorbed in a material volume of a certain length. A low $R_{T E}$ (high $\lambda$ ) and low $C_{T E}$ 
(small $C_{p}$ ) provides lower modification of the initial ohmic current and hence maximize the cooling effect. This translates into a higher value of the thermal diffusivity. However, this will make a change in the TE coefficients, so all these events described in the low frequency part of the impedance analysis reflect the common issue in the TE field where all the parameters governing the performance of the devices are interrelated.

\section{Experimental results}

Fig. 2 shows the impedance measurements of the TE module in vacuum. A semicircle is observed in the low frequency part of the Nyquist plot (Fig. 2a) that can be fitted to a parallel combination of a resistance and a capacitor. The inset of the graph shows a zoom of the high frequency response, where the intercept with the real axis provides the ohmic resistance of the module [8], $\mathrm{R}=4.0 \Omega$, which directly relates to the materials conductivity using the geometric parameters of the module. A value $\sigma=0.95 \times 10^{5} \mathrm{~S} / \mathrm{m}$ is obtained, in agreement with the value provided by the manufacturer (Table 1). 

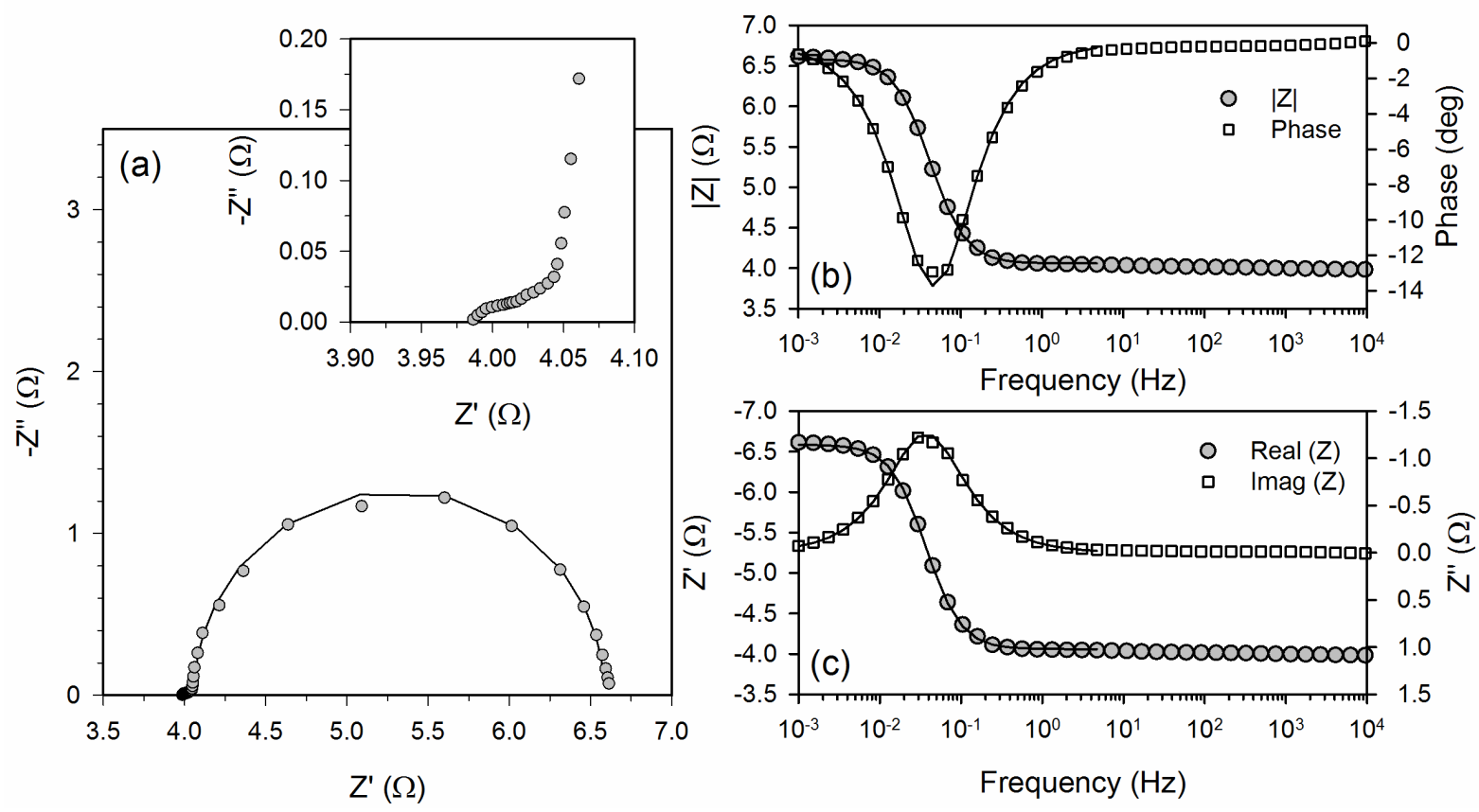

Figure 2 Nyquist (a) and bode (b, c) plots of the impedance measurements of a thermoelectric module operating as a Peltier cooler (dots and squares). Lines represent the fitting to the low frequency part. The inset on the Nyquist plot indicates a zoom to the high frequency region.

The fitting results provide a value of $R_{T E}=2.53 \Omega, C_{T E}=1.70 \mathrm{~F}$ and hence $\tau=4.30 \mathrm{~s}$. Using the values of TE properties provided in Table I, neglecting the Joule effect and taking into account that the module is formed by $254 \mathrm{legs}$, we obtain a value of $R_{T E}=3.05 \Omega, C_{T E}=0.65 \mathrm{~F}$ and $\tau=1.98 \mathrm{~s}$ which lie in the same order of magnitude than the ones obtained from the impedance fitting. We should remark that these values are obtained from parameters related to the TE materials, and the contact and ceramic plates of the module has not been taken into account. Since $\lambda$ is much higher for the contacts and the ceramic plates than in the materials, good agreement is found in $R_{T E}$. However, the specific heat of the ceramic and $\mathrm{Cu}$ contacts are in a similar order of magnitude to the $\mathrm{Bi}_{2} \mathrm{Te}_{3}$ legs, which leads to a less closer value for $C_{T E}$ and $\beta$ than the ones provided by the impedance, but in the same order of magnitude. The parameters extracted for the IS spectrum are the parameters for the whole device and hence, consideration of the contacts and ceramic plates is required for a more accurate agreement $[8$, $14]$. 
Table I Parameters for the bismuth telluride legs of the module, obtained from the module manufacturer and reference [15] when indicated by *.

\begin{tabular}{ccccccccc}
$\sigma(\mathrm{S} / \mathrm{m})$ & $\alpha(\mu \mathrm{V} / \mathrm{K})$ & $\pi(\mathrm{V})$ & $\mathrm{A}\left(\mathrm{cm}^{2}\right)$ & $\mathrm{L}(\mathrm{cm})$ & $\rho\left(\mathrm{g} / \mathrm{cm}^{3}\right)$ & $\mathrm{c}_{\mathrm{p}}(\mathrm{J} / \mathrm{gK})$ & $\lambda(\mathrm{W} / \mathrm{mK})$ & $\beta\left(\mathrm{cm}^{2} / \mathrm{s}\right)$ \\
\hline $1.04 \times 10^{5}$ & \pm 200 & \pm 0.06 & 0.01 & 0.15 & 7.7 & $0.17^{*}$ & $1.5^{*}$ & $0.007^{*}$ \\
\hline
\end{tabular}

\section{Conclusions}

The low frequency region of the impedance spectra of commercial TE modules operating as Peltier coolers has been analysed and a theoretical background developed. A new concept of a TE capacitance and a TE resistance has been introduced that accounts for the low frequency semicircle. Both $C_{T E}$ and $R_{T E}$ directly relate to the Seebeck and Peltier coefficients. The $C_{T E}$ depends on the specific heat and density of the materials and $R_{T E}$ is proportional to the reciprocal of the thermal conductivity. The time constant defined by the product of both elements directly provides the thermal diffusivity. These results provide a theoretical background for the analysis of the low frequency part of impedance results and show how all the relevant TE parameters can be obtained from a single IS measurement.

\section{Acknowledgements}

The authors wish to acknowledge financial support from the Accelerated Metallurgy Project, which is co-funded by the European Commission in the 7th Framework Programme (contract NMP4-LA-2011-263206), by the European Space Agency and by the individual partner organizations. Dr. Upul Wijayantha and Dr. Matt Carnie are gratefully acknowledged for kindly allowing taking some initial measurements in their labs. We thank European Thermodynamics Ltd. for providing the TE module used and Ahmad Akbari R. for mathematical support. 


\section{References}

1. F. Fabregat-Santiago, G. Garcia-Belmonte, I. Mora-Sero and J. Bisquert, Phys. Chem. Chem. Phys. 13, 9083 (2011)

2. X. Z. Yuan, H. J. Wang, J. C. Sun and J. J. Zhang, Int. J. Hydrog. Energy 32, 4365 (2007)

3. B. Klahr, S. Gimenez, F. Fabregat-Santiago, J. Bisquert and T. W. Hamann, J. Am. Chem. Soc. 134, 16693 (2012)

4. F. Huet, J. Power Sources 70, 59 (1998)

5. S. Dilhaire, L. D. Patino-Lopez, S. Grauby, J. M. Rampnoux, S. Jorez, W. Claeys and Ieee, In XXI International Conference on Thermoelectrics, Proceedings ICT '02, (IEEE: New York, 2002), pp 321.

6. A. D. Downey and T. P. Hogan, In ICT2005, 24th International Conference on Thermoelectrics, (IEEE: New York, 2005), pp 79.

7. A. D. Downey, E. Timm, P. F. P. Poudeu, M. G. Kanatzidis, H. Shock and T. P. Hogan, In Materials and Technologies for Direct Thermal-to-Electric Energy Conversion, ed. J. Yang, Hogan T. P., Funahashi R. and Nolas G. S. (Materials Research Society: Warrendale, 2006), pp 425.

8. A. D. Downey, T. P. Hogan and B. Cook, Rev. Sci. Instrum. 78, 93904 (2007)

9. E. Hatzikraniotis, K. T. Zorbas, I. Samaras, T. Kyratsi and K. M. Paraskevopoulos, J. Electron. Mater. 39, 2112 (2010)

10. A. De Marchi and V. Giaretto, Rev. Sci. Instrum. 82, 104904 (2011)

11. D. E. Wesolowski, R. S. Goeke, A. M. Morales, S. H. Goods, P. A. Sharma, M. P. Saavedra, K. R. Reyes-Gil, W. C. G. Neel, N. Y. C. Yang and C. A. Apblett, J. Mater. Res, 27, 1149 (2012)

12. H. J. Goldsmith, Electronic refrigeration, (Pion, London, 1986).

13. R. R. Heikes and R. W. Ure, Thermoelectricity: science and engineering, (Interscience, New York, 1961).

14. A. De Marchi and V. Giaretto, Rev. Sci. Instrum. 82, 104904 (2011)

15. H. Wang, W. D. Porter, H. Bottner, J. Konig, L. D. Chen, S. Q. Bai, T. M. Tritt, A. Mayolet, J. Senawiratne, C. Smith, F. Harris, P. Gilbert, J. Sharp, J. Lo, H. Kleinke and L. Kiss, J. Electron. Mater. 42, 1073 (2013) 